\title{
All-optical Read Only Memory Employing SOAs
}

\author{
Young Jin Jung and Namkyoo Park \\ School of EECS, Seoul National University, 599 Gwanangno, Gwanak-gu, \\ Seoul, 151-742, South Korea \\ Young Min Jhon and Seok Lee* \\ Korea Institute of Science and Technology, 5 Wolsong-gil, Seongbuk-gu, \\ Seoul, 136-791, South Korea
}

(Received January 7, 2008 : revised February 8, 2008)

\begin{abstract}
An all-optical read only memory utilizing cross gain modulation in semiconductor optical amplifiers (SOAs) has been demonstrated for the first time to our knowledge. In our demonstration, an all-optical 2-to-4 line decoder constructed with SOAs has been employed for the construction of this all-optical read only memory. Storing four characters in an American standard code for information interchange (ASCII) format has been successfully carried out. Each character consisting of seven binary bits could be read out at a rate of 10 Giga characters per second.

OCIS codes : 250.5980, 200.3760, 200.4660, 070.4560, 200.4540
\end{abstract}

\section{INTRODUCTION}

Every stored-program computer requires non-volatile memory to save the initial program that runs when the computer is turned on or otherwise begins execution. The memory used for this purpose is not necessarily rewritable, so read-only memory (ROM) can be used for saving the initial program. Usually it is cost effective to use ROM rather than random access memory (RAM), so many computers employ ROM to store the initial program. Meanwhile, much effort has been made to overcome the speed limitations of electronic circuits by constructing circuits all-optically. All of the all-optical Boolean logic gates have been demonstrated [1-5] by means of cross gain modulation (XGM), and XGM-based logic gates have attracted attention for their simple structures compared with cross phase modulation (XPM)based logic gates, although the speed and the extinction ratio are somewhat worse for XGM-based logic gates than for XPM-based logic gates. However, all-optical ROM has not been demonstrated yet even with other kinds of mechanisms including XPM due to the complexity and immature technology of all-optical circuit elements. Thus, study of the all-optical ROM is worthwhile because of its potential to overcome the speed limitations of electronic circuits.

In this research, we construct all-optical ROM where four characters are stored in the American standard code for information interchange (ASCII) format utilizing an all-optical 2-to-4 line decoder. First, experimental demonstrations of the Boolean operations that are required to construct an all-optical 2-to4 line decoder are carried out, and then an all-optical ROM employing this decoder is demonstrated using a numerical assessment.

\section{THEORY}

The all-optical ROM that we would like to demonstrate is the ROM where four characters are stored in a form of ASCII code, so we stored the word 'KIST' in the ROM as shown in Figure 1. (The word 'KIST' is just an example for the demonstration of this system, so this word can be changed by using other characters that can be formed by changing some of the connections and this will be illustrated later.) The ASCII code table for each of the characters in 'KIST' is shown in Table 1. When the inputs of the 2-bit address are applied to this ROM, the corresponding outputs of the 7-bit ASCII character come out.

In order to implement this function, we utilize the 2-to-4 line decoder that is common in digital electronics [6]. The function of the 2-to-4 line decoder is illustrated in Figure 2 with the truth table that shows its Boolean operations.

If we let the input be a binary number where $I_{1}$ is 


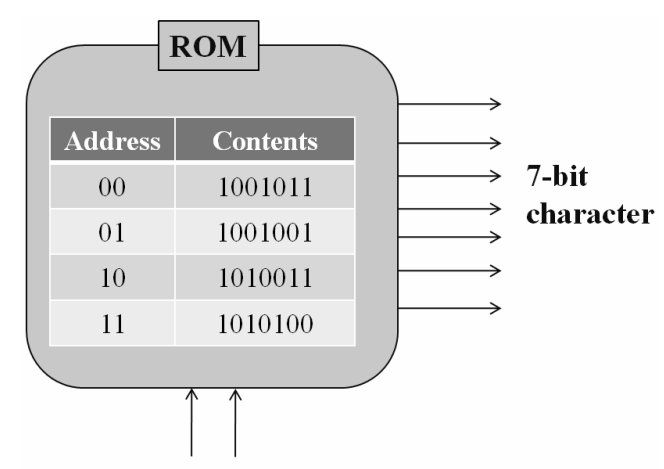

2-bit address

FIG. 1. Schematic representation of the all-optical ROM.

TABLE 1. ASCII code table for each characters of 'KIST' as a binary number

\begin{tabular}{c|c}
\hline \hline Character & Binary \\
\hline $\mathrm{K}$ & 1001011 \\
$\mathrm{I}$ & 1001001 \\
$\mathrm{~S}$ & 1010011 \\
\hline $\mathrm{T}$ & 1010100 \\
\hline
\end{tabular}

the most significant bit (MSB) and $I_{0}$ is the least significant bit (LSB), only the output port number that corresponds to the binary number indicates level-1 while the others indicate level-0. The method of constructing a ROM with a line decoder can be understood with the help of digital electronic theory and also can be seen with later illustrations of our construction. Figure 3 shows the final goal of our demonstration where the dots at the cross points mean that there is an optical signal summation of the decoder outputs. If we designate the MSB as Bit6 and the LSB as Bito, each bit of the outputs can be written as shown in Equation (1). It is worth noting that Bit6 can be implemented by nothing but a Clock signal, but we obtain it as Equation (1-a) to observe the signal degradation. Simple logical calculations show that these output bits from the ASCII word 'KIST' in response to the four binary numbers at the inputs to the decoder.

$$
\begin{aligned}
& \text { Bit6 }=O_{0}+O_{1}+O_{2}+O_{3} \\
& \text { Bit5 }=\text { null } \\
& \text { Bit4 }=O_{2}+O_{3} \\
& \text { Bit3 }=O_{0}+O_{1} \\
& \text { Bit2 }=O_{3} \\
& \text { Bit } 1=O_{0}+O_{2} \\
& \text { Bit } 0=O_{0}+O_{1}+O_{2}
\end{aligned}
$$

To construct this function all-optically, we utilized the XGM process in SOAs. Boolean expressions of the outputs for the 2-to-4 line decoder can be written as

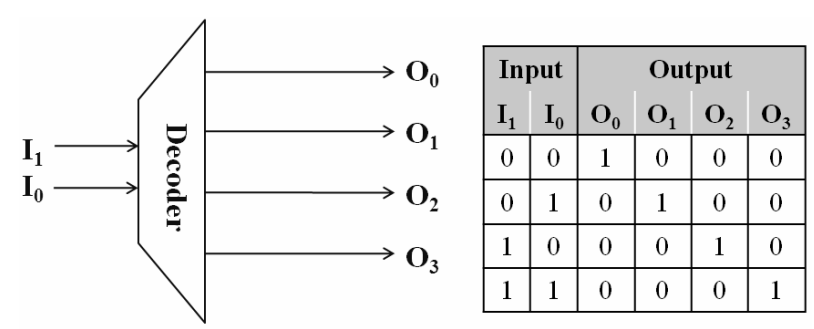

FIG. 2. The function of the $2^{-t^{-}}-4$ line decoder.

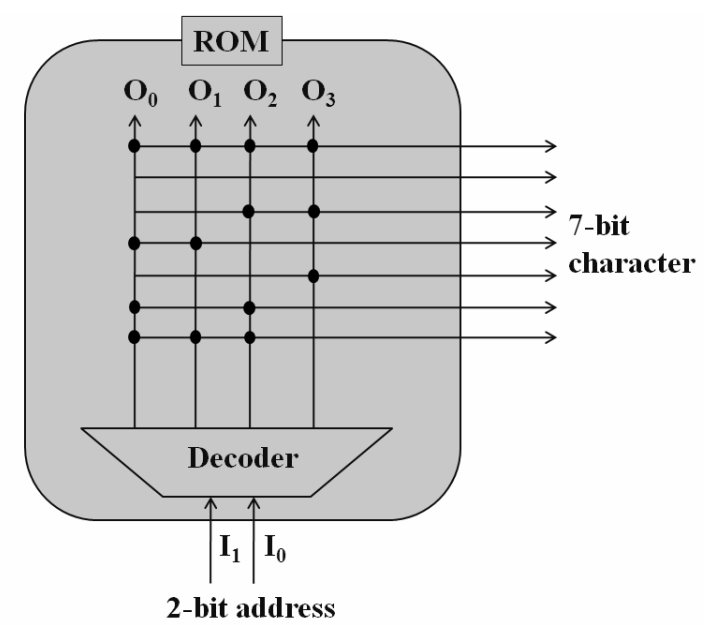

FIG. 3. The constructed all-optical ROM where four characters are stored in the ASCII code format utilizing the all-optical 2-to-4 line decoder. The word 'KIST' is stored in this schematic.

Equation (2) and its all-optical implementations are shown in the Figure 4. From Figure 4, the logical operation principles can be understood by figuring out the roles of the pump and probe signals [1-5]. The RZ signal was assumed for our implementation because the $\mathrm{XGM}$ process for the RZ signal is generally faster than the NRZ signal according to [5]. This is because the guard time for the carrier recovery can be guaranteed. Furthermore, the RZ signal has the advantage of hiding the static " 1 " hazard compared to the NRZ signal [6].

$$
\begin{aligned}
& O_{0}=\overline{I_{1}} \overline{I_{0}} \\
& O_{1}=\overline{I_{1}} I_{0} \\
& O_{2}=I_{1} \overline{I_{0}} \\
& O_{3}=I_{1} I_{0}
\end{aligned}
$$

\section{RESULTS}

The demonstration of the 2-to-4 line decoder was carried out with experiments for the specific input patterns making all of the possible one-zero combinations. In 


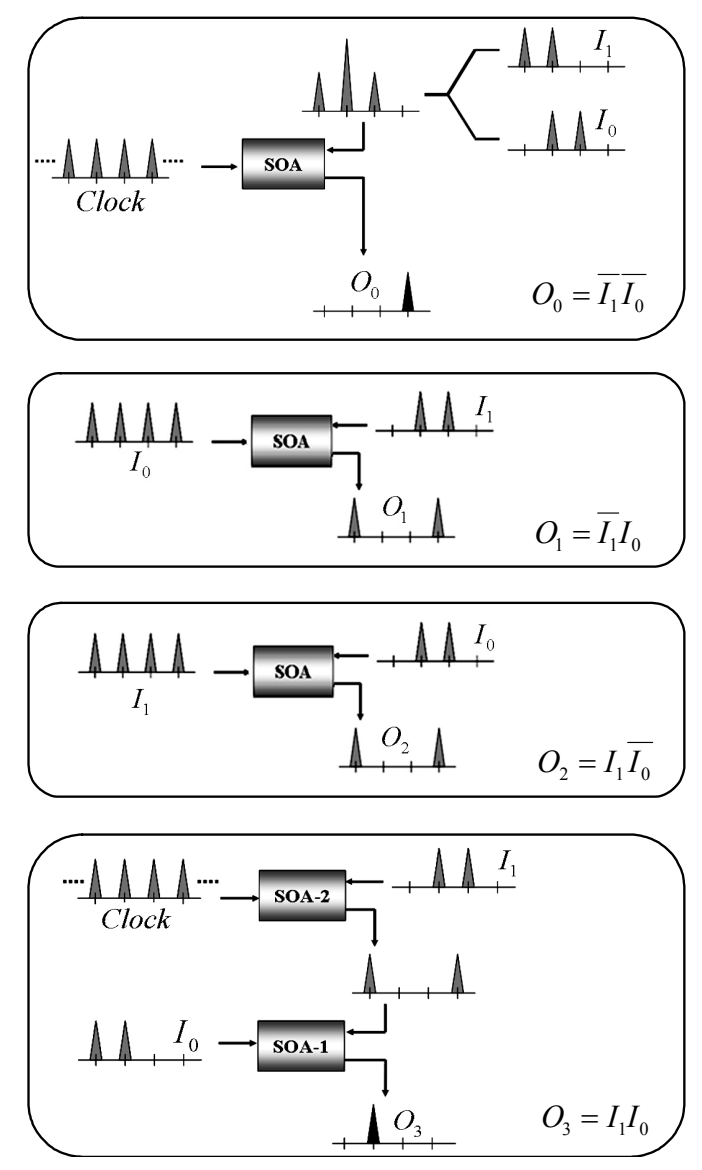

FIG. 4. All optical implementations of each output of the 2-to-4 line decoder utilizing XGM in SOAs.

order to optimize the XGM process, attenuators and erbium doped fiber amplifiers (EDFAs) were used to adjust the pump and probe power levels. $10 \mathrm{Gbps}$ RZ input patterns were applied to the 2-to-4 line decoder and the exact output patterns were obtained as shown in Figure 5.

As it is difficult to make independent random input patterns of $I_{1}$ and $I_{2}$ and experimental demonstration is somewhat bulky, the operation of the all-optical ROM and the signal quality evaluations were carried out using a numerical assessment. In our numerical assessment, the propagation equation and the rate equation were adopted from reference [7] to create Equations (3) and (4). To make faster calculations, the integral equation approach [8] was used for the static simulation by modifying Equation (3), so the equation takes the integral form as shown in Equation (5). The SOA simulation parameters that correspond to Equations (3)-(5) are shown in Table 2 with the parameter names.

$$
\frac{d a_{l}}{d z}=\frac{1}{2} g(N)\left[\sum_{m=c p, s h b, c h} \frac{(1-j \alpha) a_{l}-}{1+j \Delta w_{i j} \tau_{m}} a_{i} a_{j}^{*} a_{j} a_{k}\right]-\frac{\gamma_{s c} a_{l}}{2}
$$
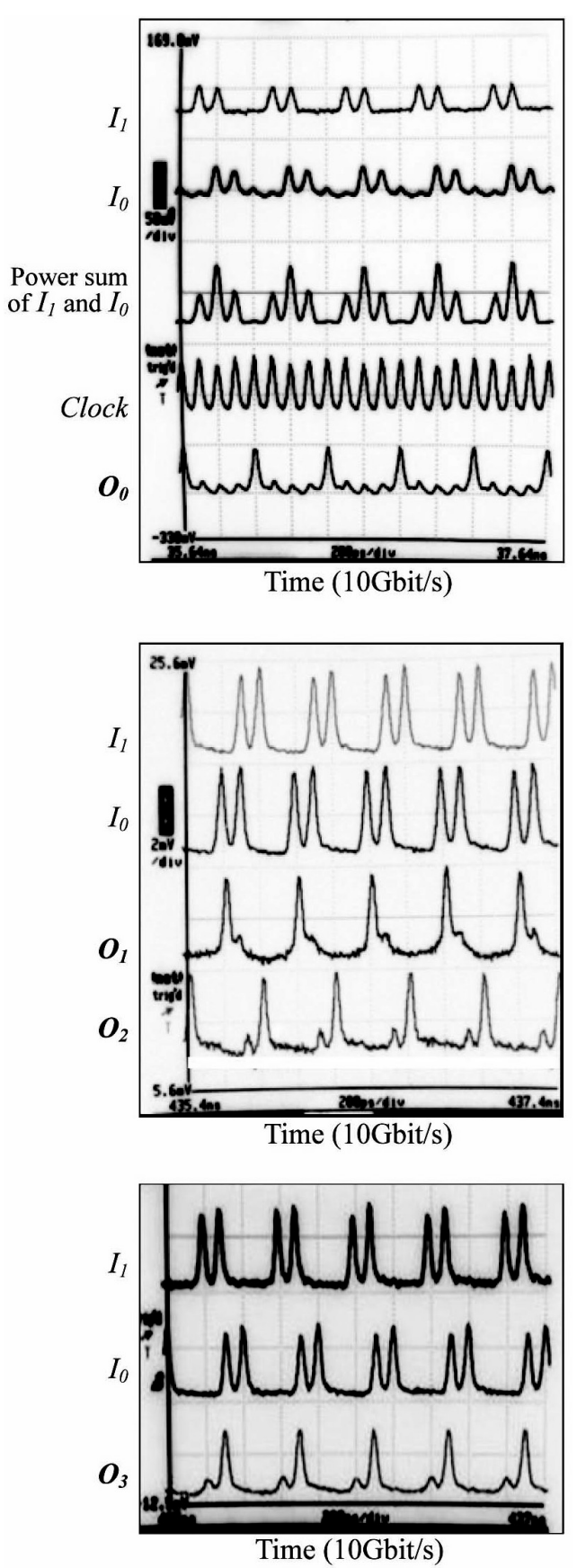

FIG. 5. Experimental results of the all-optical 2-to-4 line decoder.

$$
\begin{aligned}
& \frac{d N}{d t}=\frac{J}{q d}-\frac{N}{\tau_{s}}-\frac{g(N)}{\hbar \omega_{0}}|E|^{2}, \quad \tau_{s}=\frac{1}{A+B N+C N^{2}} \\
& a_{l}(z)=a_{l}(0)+\int_{0}^{2} \frac{1}{2} g_{l}(N)\left[-\sum_{m}\left(\sum_{\Delta o_{i}} \frac{\left(1-j \alpha \beta_{m}\right) \varepsilon_{m}}{1+j \Delta \omega_{i j} \tau_{m}} a_{i}^{*} a_{j} a_{k}\right)\right]-\frac{\gamma_{s c} a_{l}}{2} d z
\end{aligned}
$$

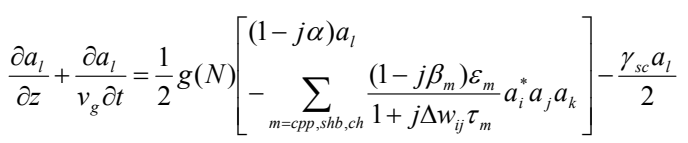


TABLE 2. Names and values of SOA simulation parameters

\begin{tabular}{|c|c|c|c|c|}
\hline$a_{l}(z)$ & \multicolumn{4}{|c|}{ complex amplitudes of the signal fields } \\
\hline$z$ & \multicolumn{4}{|l|}{ propagation axis } \\
\hline$N$ & \multicolumn{4}{|l|}{ carrier density } \\
\hline$g(N)$ & \multicolumn{4}{|l|}{ modal gain } \\
\hline$a$ & \multicolumn{3}{|c|}{ linewidth enhancement factor } & 10 \\
\hline$\gamma_{s c}$ & \multicolumn{3}{|c|}{ scattering loss per unit length } & $34 \mathrm{~cm}^{-1}$ \\
\hline ' $i, j, k, l$ ' & \multicolumn{4}{|c|}{ index of different wavelengths } \\
\hline$\Delta \omega_{i j}$ & \multicolumn{4}{|c|}{ frequency difference $\left(\omega_{i}-\omega_{j}\right)$} \\
\hline$\varepsilon_{m}$ & \multicolumn{2}{|c|}{$\begin{array}{l}\text { inverse saturation powers } \\
\text { from the nonlinearity }\end{array}$} & \multicolumn{2}{|c|}{$\begin{array}{l}\text { shb: } 0.91 \mathrm{~W}^{-1}, \\
\text { ch: } 1.62 \mathrm{~W}^{-1}\end{array}$} \\
\hline$\beta_{m}$ & \multicolumn{2}{|c|}{$\begin{array}{l}\text { contributions of line-width } \\
\text { enhancement factor }\end{array}$} & \multicolumn{2}{|c|}{$\begin{array}{ll}\text { shb: } 0.21 & \mathrm{~W}^{-1}, \\
\text { ch: } 2.812 & \mathrm{~W}^{-1}\end{array}$} \\
\hline$\tau_{m}$ & \multicolumn{2}{|c|}{ relaxation times } & \multicolumn{2}{|c|}{$\begin{array}{l}s h b: 0.036 \mathrm{ps}, \\
s h b: 0.52 \mathrm{ps}\end{array}$} \\
\hline index $m$ & \multicolumn{4}{|c|}{$\begin{array}{l}\text { carrier population pulsation }(c p p) \\
\text { spectral hole burning }(s h b) \\
\text { and carrier heating }(c h)\end{array}$} \\
\hline$J$ & \multicolumn{4}{|l|}{ current density } \\
\hline$q$ & \multicolumn{4}{|l|}{ electron charge } \\
\hline$d$ & \multicolumn{4}{|c|}{ active layer density } \\
\hline$A, B, C$ & $\begin{array}{l}\text { Recombination } \\
\text { coefficient }\end{array}$ & \multicolumn{3}{|c|}{$\begin{array}{l}\text { A: } 1 \times 10^{8} \mathrm{~s}^{-1} \\
\text { B: } 2.5 \times 10^{-11} \mathrm{~cm}^{3} \mathrm{~s}^{-1} \\
\text { C: } 1 \times 10^{-28} \mathrm{~cm}^{6} \mathrm{~s}^{-1}\end{array}$} \\
\hline
\end{tabular}

For the time domain dynamic simulations, Equation (3) was expanded as a full propagation equation including the time dependence as shown in Equation (6), and the transfer matrix method [9] was utilized with Equation (6). Taking SOAs that have lengths of $300 \mu \mathrm{m}$ and driving currents of $300 \mathrm{~mA}$, the XGM processes were optimized by adjusting the pump and probe power levels with the help of the static simulation results shown in Figure 6 . Pump power levels of $0 \mathrm{dBm}$ to $10 \mathrm{dBm}$ and probe power levels of $-20 \mathrm{dBm}$ to $-10 \mathrm{dBm}$ were assumed for the 0-level to 1-level in the analysis. Amplified spontaneous emission (ASE) noise was added to both the input signals and the clock signal, so all of the input signals have an OSNR of around $17 \mathrm{~dB}$.

Figure 7 shows the simulation results of the all-optical ROM. Decoder output signals from $\mathrm{O}_{0}$ to $\mathrm{O}_{3}$ agreed well with our previous experiment shown in Figure 5. For each input address from $0\left(I_{1}=0, I_{0}=0\right)$ to $3\left(I_{1}\right.$ $\left.=1, I_{0}=1\right)$, the corresponding output ASCII code characters were achieved exactly from ' $\mathrm{K}$ ' to ' $\mathrm{T}$ ' as expected. As can be seen in Figure 7 and Figure 8, the zero level of the output bit was more floated as many decoder outputs were added. The signal quality was evaluated by calculating the eye diagram with a $2^{7}$-bit

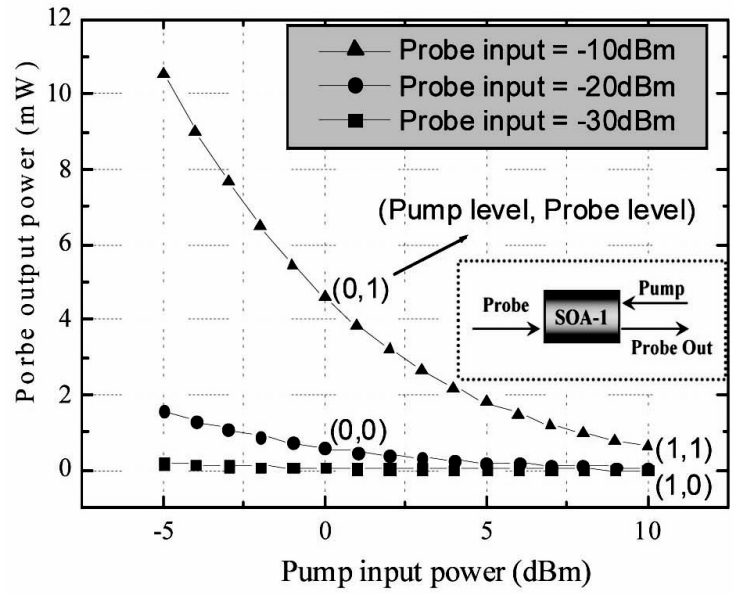

FIG. 6. Simulated XGM characteristics of the steady state response of a SOA; Only the case of '(Pump level, Probe level $)=(0,1)$ ' makes the output level “1”, while others make the level " 0 ".

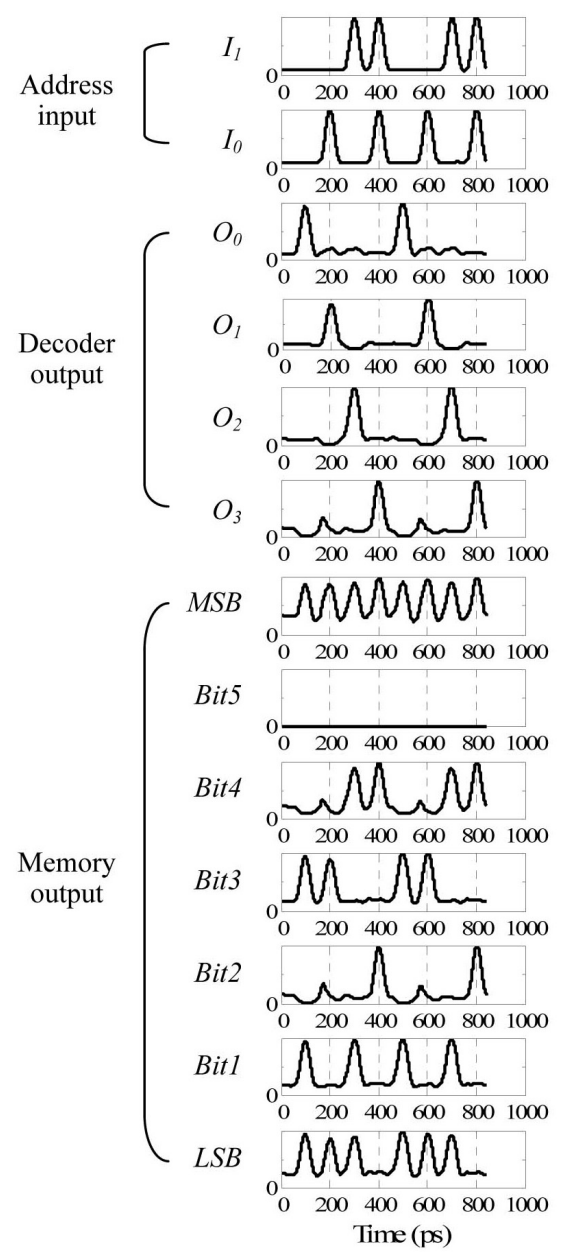

FIG. 7. Simulation results of patterns for the all-optical ROM.

pseudo random bit sequence (PRBS) input pattern that takes different seed values. Eye diagrams from Bit0 


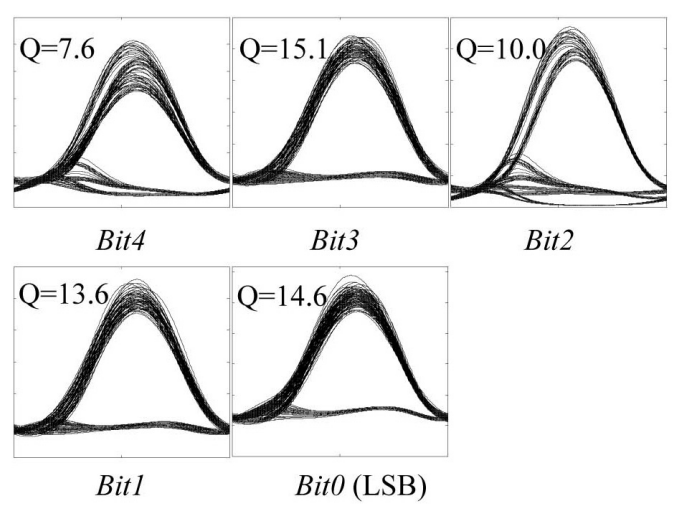

FIG. 8. Simulation results of eye-diagrams for the alloptical ROM. A 127 bit PRBS was applied for each input with different seed values.

(LSB) to Bit4 are shown in Figure 8. (It was not necessary to evaluate the qualities of Bit5 and Bi6 (MSB) because these bits do not contain any information in the case of the word 'KIST'.) Q factors of above 7 could be achieved for all of the output values.

\section{CONCLUSION}

We have demonstrated an all-optical ROM employing an all-optical 2-to-4 line decoder based on XGM in SOAs. Experimental demonstrations of the decoder output have been carried out and a numerical study for storing the four characters on the ROM has been done. Q factors of above 7 could be achieved with input signals having a $17 \mathrm{~dB}$ OSNR while running at 10 Gbps.

*Corresponding author: slee@kist.re.kr

\section{REFERENCE}

[1] J. H. Kim, B. C. Kim, Y. T. Byun, Y. M. Jhon, S. Lee, D. H. Woo, and S. H. Kim, "All-optical AND gate using cross-gain modulation in semiconductor optical amplifiers," Japanese J. Appl. Physics, vol. 3, no. 2, pp. 608-610, 2004

[2] Y. T. Byun, K. S. Choi, Y. M. Jhon, D. H. Woo, S. Lee, S. H. Kim, and J. W. Park, "All-optical OR gate using cross gain modulation in semiconductor optical amplifier,” CLEO Europe, pp. 493, June. 2005.

[3] J. H. Kim, Y. M. Jhon, Y. T. Byun, S. Lee, D. H. Woo, and S. H. Kim, "All-optical XOR gate using semiconductor optical amplifiers without additional input beam," IEEE Photonics Technology Letters, vol. 14 no. 10, pp. 1436-1438, 2002.

[4] S. H. Kim, J. H. Kim, B. G. Yu, Y. T. Byun, Y. M. Jeon, S. Lee, D. H. Woo, and S. H. Kim, "All-optical NAND gate using crossgain modulation in semiconductor optical amplifiers," IEE Electronics Letter, vol. 41, no.18, pp. 1027-1028, 2005.

[5] Y. T. Byun, J. H. Kim, Y. M. Jhon, S. Lee, D. H. Woo, and S. H. Kim, "High-speed all-optical NOR gate using semiconductor optical amplifier," CLEO Europe, pp. 536, Jun. 2003.

[6] R. H. Katz and G. Borriello, Contemporary Logic Design, 2nd ed. (Prentice Hall, 1993).

[7] M. A. Summerfield, and R. S. Tucker, "Frequency domain model of multiwave mixing in bulk semiconductor optical amplifiers," IEEE J. Sel. Top. Quantum Electron, vol. 5, no. 3, 839-850, 1999.

[8] Y. J. Jung, P. Kim, J. Park, and N. Park, "Integral equation approach for the analysis of high-power semiconductor optical amplifiers," Optics Express, vol. 14, no. 6, pp. 2398-2403, 2006.

[9] M. G. Davis and R. F. O’Dowd, “A transfer matrix method based large signal dynamic model for multielectrode DFB lasers," IEEE J. Quantum. Electron., vol. 30, no. 11, pp. 2458-2466, Nov. 1994. 